\title{
DE LA PERSPECTIVA GRAMATICAL A LA PERSPECTIVA COMUNICATIVA
}

\author{
(A propósito del libro Discurso y desarrollo de la competencia en la educación \\ básica, de los profesores Justo Morales Álvarez y Maria Teresa Cortés)
}

\author{
RUBÉN ARBOLEDA TORO \\ Universidad Nacional \\ de Colombia abril. 1997
}

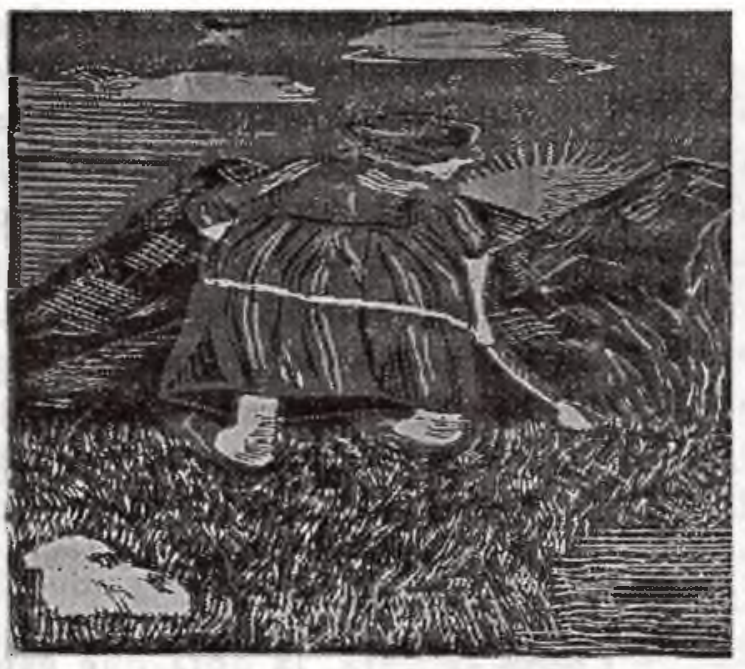

principio del autor o del lector tomados independientemente; contituye simplemente el encuentro o desencuentro inicial de ellos. Con el libro de Justo y María Teresa eperimenté un desencuentro inicial: esperaba una sistematización de experiencias de desarrollo de la competencia comunitiva en la escuela formal. Pero ¿Por qué esperaba una sistematización? En principio, porque estoy acostumbrado a ese típo de obras, porque corresponde a la clase de obras que he escrito. Pero este

Agradezco a los autores que me hayan elegido para esta presentación. Obedece ella seguramente a mi labor en el campo del cultivo de la lengua materna. Quiero hacerme a la idea que con el programa de Español y Comunicación Educativa desde cuando se inició como Lingüística y Literatura desde cuando emprendió el promisorio proyecto Atlas Lingüístico del Huila, que a mi modo de ver mantiene rasgos de validez y necesariedad, aún en su relativo olvido, como se presiente desde otros proyectos sociodialectales y aún pedagógicos en marcha, en torno de los cuales valdría la pena dialogar en momentos más pertinentes.

Normalmente un lector se acerca a un libro con una expectativa o como dicen con una hipótesis de lectura, cuya comprobación o disprobación nada dice en hecho nada dice valorativamente del libro en sí mismo; corresponde simplemente nuestra primera relación, la del libro y yo, una relación de desconcierto, que llegó a ser armónica en la medida que me dediqué a comprender el libro que tenía entre manos, a descubrirlo, y no el que mi predisposición esperaba.

Fue como cuando en una esquina se espera a alguien y de pronto se nos pone en frente una persona que no es la esperada, y después del desconcierto inicial se establece con ella una comunicación gratificante y el gusto empieza a llegar, en cuanto se va encontrando la especificidad de la persona traída por las circunstancias.

Esta acción de comprensión, de descubrimiento del otro y de lo otro en sí mismo, es uno de los fundamentos de la 
comunicación ética, de la comunicación para el desarrollo humano, a la que tanto han hecho referencia nuestro amigo Leonel Arias y los autores del libro que comento. Y no se trata de un encuentro del otro en sí mismo para la complacencia, como simplistamente podría pensarse, sino de una comprensión del otro que potencie una dialógica, una contrastación sustentada en el entendimiento, en la aceptación del otro en cuanto congénere, ni más ni menos que en cuanto ser que comparte conmigo la especie a la cual se pertenece.

En todo caso, el encuentro con el libro de María Teresa y Justo evidencia la convivencia, la coexistencia de dos tipos de obras, en este caso en relación con proyectos educativos: aquella que va presentando la sistematización de las acciones, de las experiencias pedagógicas específicas, de los métodos, estrategias o procedimientos empleados, en una especie de trama, tejido o urdimbre con las ideas, conceptos, convicciones e incluso creencias, intuiciones, por qué no, en las cuales se fundamentan las acciones, y que constituyen su marco o su perspectiva teórica. Y otra de categorización más fuerte, en el sentido bernsteiniano, de mayor explicitud hipotético-deductiva, en la cual los autores optan por presentar el marco teórico, de manera integral, luego el componente metodológico o procedimental y finalmente las acciones derivadas. Esto por supuesto no significa que en la contrucción de este tipo de obra no haya un ir y venir permanente entre el marco teórico y la aplicación, así como la sustitución comprensiva de prácticas puede originar cambios en la conceptualización o teorización de la acción, en una dialéctica recurrente. Tal vez podría pensarse en dos polos en medio de los cuales se mieven obras de diferente predominancia, a la manera como los textos realizan distintas funciones del lenguaje en medio de una predominate, según lo señala Roman Jakobson en su clásico ensayo Lingüística y poética.

Por supuesto no estoy postulando procederes mejores o peores sino reconociendo modelos o modalidades distintas. Para ciertos estudios puede resultar más cómoda la primera; para otros, la segunda. Pero aderiás del objeto, la opción tiene que ver con los estudiosos. Unos se desenvuelven mejor en la modalidad que podríamos denominar testimonial (más cercana a los etnográfico), establecen con ella una buena relación cognitiva y afectiva; otros se interesan más por la delimitación y explicitación de las fases del proceso hipotético-deductivo. sucede incluso el tránsito de una a otra. Tampoco estas modalidades están vinculadas a un mayor o menor rigor; el rigor tiene que ser un rasgo característico de una y otra. Obviamente siempre será deseable que la divulgación de los avances teóricos sea tan clara como lo permita el objeto.

Como se verá, la obra que estoy comentando se inclina hacia la segunda modalidad, la de categorización más fuerte. Los autores inician la obra con este razonamiento deductivo: todo grupo social desarrolla modos de comunicación para entenderse e interactuar en las actividades diarias. Parte del desarrollo humano de los pueblos depende entonces de que la interacción social comunicativa de calidad. Ante esto, las naciones y pueblos tienen la obligación de ofrecer a los niños y jóvenes una educación de calidad, favorable a una interacción social comunicativa de calidad. En este sentido, la enseñanza de la lengua materna (cultivo, diría yo) es de calidad si desarrolla al máximo la competencia comunicativa social, si cumple el objetivo de "capacitar a los niñ̈os en :: producción y comprensión de discursos en su lengua materna, sobre el conocimiento del mundo natural, las experiencias de la vida 
cotidiana, la interacción social, y la participación en la construcción de una nueva sociedad civil, más digna y más humana que la presente". (pp. 13-15). Si logra, como lo expresan los autores en las dos últimas páginas del libro, que el niño no sólo esté en capacidad de reconstruir el signifcado global y los diferentes niveles de coherencia de un discurso, sino de "construir criticas sociales, expresar opiniones, tener actitudes propias y colectivas, aceptar valora ciones, orientar modos nuevos de convivencia social y solidaridad social, etc., frente al significado comunicativo de los textos leídos/escuchados".

En una clasificación gruesa e indiscriminada, los autores afirman que en la enseñanza de la lengua materna en nuestro país se ha presentado tres orientaciones: la tradicio nal, la formal y la semántico comunica tiva; en una conside ración evaluativa general concluyen que en ninguno de ellos se ha cumplido satisfactoriamente el propósito de desarrollar la competencia comunicativa social de los niños. Para lograr el objetivo, proponen entonces el Paradigma de la Interacción Social Comunicativ, por oposición al que denominan paradigma gramatical o formal, en el cual se inscriben, en su opinión, los tres momentos mencionados.
Piensan los autores que para poder construir las estrategias comunicativas, las estrategias discursivas que se pondrán en marcha en los centros educativos en función del objetivo mencionado, es necesario establecer los principios teóricos y metodológicos (perspectivas teóricas y metodológicas, dicen los autores para limitar su alcance) en que se fundamentará dicha construcción. Al establecimento de esos principios le han dedicado tiempo y esfuerzo. Justamente los resultados se recogen en el libro que presentamos. A partir de dichas perspectivas los educadores podemos construir estrategias, insisten. De ahí la importancia del libro. Pero además. los autores vienen construyendo lo que denominan estrategias comunicativas de producción de discurso, que piensan presentar en un segundo libro; entre otras, mencionan estrategias comunicativas para la generación del pensamiento lógico, la formación de la conciencia crítica, la elaboración cognitiva de la información, la elaboración o recepción de la comunicación masiva y estrategias para la crítica social del discurso.

Los autores reconocen en la historia de la lingüistica dos tipos de estudio del lenguaje: los estudios gramaticales o 
formales frente a los estudios comunicativos del lenguaje. Y puntualmente afirman que las perspectivas teóricas para el desarrollo de la competencia comunicativa social de los niños se encuentran en los estudios comunicativos. Ubican acá estudios como los de Vigotski. Mead y Sapir, que desde los años veinte se interesaban por entender el lenguaje en el marco de la comunicación, la actividad humana, la generación del pensamiento y el contexto cultural, que en gran medida fueron opacados después de la segunda guerra mundial, afirman, por los estudios gramaticales y formales, concentrados en la estructura interna de las lenguas. especialmente en sus niveles fonológico y morfosintáctico. Y por supuesto ubican en el marco comunicativo, los extraordinarios avances que sobre todo desde los años sententa han ido alcanzando la sociolingüistica, la psicolingüistica, la semántica, la pragmática y la teoría del discurso. principalmente, en las cuales emerge de nuevo la perspectiva comunicativa y sociocultural.

Entonces Justo y María Teresa compendian y adecúan en su libro el conocimiento más decantado de estos estudios comunicativos y el más pertinente para orientar el desarrollo de la competencia comunicativa social de los niños (caps. 1 y 3). Labor ardua la de los autores. Resalta en el libro el compendio y adecuación de la teoría del discurso. En el primer capítulo, sus cinco dimensiones: cognitiva, comunicativa, semántica, pragmática y sociocultural. En el tercero. fundamentos teóricos sobre intención comunicativa, coherencia semántica, referentes, actores, microestructura semántica, macroestructura semánticopragmática, superestructura esquemática, elaboración cognitiva de la información, generación del pensamiento lógico, estructura superficial y formación de la conciencia crítica. A manera de ejemplo destaco la sistematización de las conceptualizaciones acerca de los actos ilocucionarios, en un recorrido desde Austin hasta Vanderveken (pág. 63). También, la manera didática como recurren a las formas de acción tipificadas en la realización de un aseo personal diario, para que se comprendan mejor los procesos de institucionalización, legitimación y socialización en los cuales interviene el lenguaje de manera decisiva (págs. 40ss).

Se adivina, pero también se insinúa en el libro, el espíritu que ha orientado la labor universitaria de los autores, de la cual deriva aquel: un acercamiento detenido a la teoría, en este caso del discurso, y, en consonancia con los profesionales en cuya formación intervienen, una búsqueda de proyecciones en la docencia. Hablan pues en el libro dos estudiosos de la lingüística, muy especialmente de la teoría del discurso, que en la acción educativa ha comprendido la significación del desarrollo de la competencia comunicativa social de los hablantes. Es entonces muy elocuente el título del libro: Discurso y desarrollo de la competencia comunicativa en la educación básica.

Como era de esperarse tratándose de un conocimiento tan vasto, algunas partes del compendio son apretadas, lo cual oscurece un tanto los contenidos; pero la referencia continua a la bibliografía ubica al lector y lo invita a remitirse a ella. A propósito, es notable la riqueza bibliográfica: amplia, pertinente, pluridisciplina y actualizada. Por supuesto los conocimientos compendiados podrían ser objeto de análisis detallado, pero dicha tarea lingüistica desborda los límites de este comentario.

En el capítulo 2 explicitan perspectivas metodológicas inspiradas en dicho conocimiento, razón por la cual lucen muy sugerentes y prometedoras para la 
construcción de las estrategias comunicativas de producción de discurso. Cada día se llega más al convencimiento de que a la educación formal la ha resentido la falta de una comunicación auténtica, en la cual educadores y educandos se asuman como verdaderos interlocutores en procesos de conocimientos. De ahí que destaque esta perspectiva metodológica del libro: "En consecuencia todo proceso educativo debería ser primero que todo un proceso comunicativo compartido entre maestros y niños sobre la significación, comprensión y entendimiento mutuos de esa realidad natural y social, tangible y observable que es la vida cotidiana en su continuo fluir". (p. 134).

Los autores trazan una frontera rígida entre sus enfoque para la enseñanza (cultivo, insisto) de la lengua materna y los enfoques previos ya mencionados, a tal punto que, en el marco de Kuhn, considera su propuesta un paradigma, el de la Interacción Social Comunicativa, frente al paradigma gramatical, en el cual inscribe los otros enfoques. Parece ser que el establecimiento de esta frontera responde a la aceptación de que entre la lingüistica del sistema y la lingüistica de la comunicación también existe una ruptura paradigmática (asunto que amerita una investigación).

Es esperable que el modelo lingüistico predominante en una época oriente la pedagogía de las lenguas predominantes en ella. El avance de la lingüistica de la comunicación, aún cuando coexiste con otras prácticas lingüisticas de grandes desarrollos, es de gran contundencia e impacto hoy en día. Quién sabe cómo irán a ser las cosas en un fituro. Quizas se llegue a un cultivo de las lenguas en el mundo educativo, mediado por una especie de síntesis entre la orientación sistemática y la comunicativa. La dialéctica

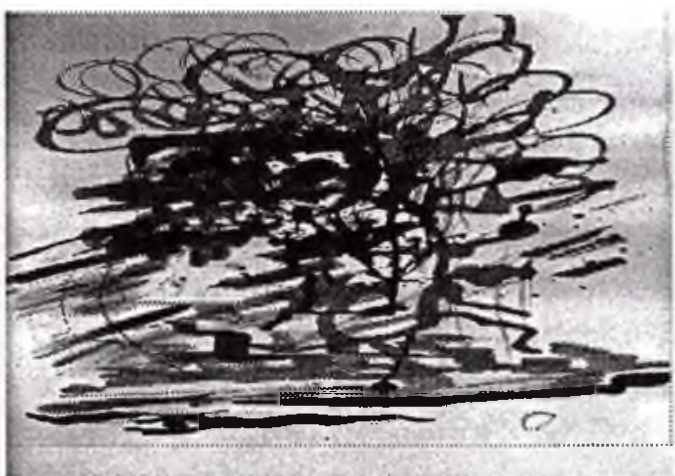

de la antítesis frente a la tesis para el arribo a una síntesis parece un imperactivo del devenir. Parecería que el detenimiento alternativo en los polos fuera una condición para el descubrimiento de puentes subyacentes. Así parece haber sido en la historia de la lingüistica. El detenimiento en la tesis saussureana de la lengua (sistema) como objeto de la lingüistica generó los extraordinarios avances de la lingüistica estructural y la generativa transformacional: pero a ese detenimiento en la lengua le siguió uno en el habla, el uso, la interacción comunicativa, de desarrollo igualmente admirables. Las síntesis de estos momentos se insinúa cada vez más en obras como la de Eugenio Coseriu y Adolfo Elizaincín. por citar dos nombres: uno europeo y uno latinoamericano; pero se insinuaba desde mucho antes, por ejemplo en $W$. $v$. Wartburg y en Henry Frei (realización del funcionalismo praguiano): y es más, en el Curso mismo de Saussure.

A lo largo del libro es constante la explicitación de los contrastes entre los paradigmas para la enseñanza y el señalamiento de las virtudes del que proponen. Los autores, muy consciente del arraigo de los estudios gramaticales o formales en el contexto educativo, aprovechan toda ocasión favorable para mostrar limitaciones de estos en cuänto perspectiva teórica para una pedagogía de la lengua tendiente al desarrollo de la competencia comunicativa social de los niños. 
Por ejemplo, la p. 21 dice que la oración es la unidad básica de análisis de los estudios gramaticales, en tanto que el discurso lo es de la dimensión comunicativa, en cuanto el discurso, no la oración, "es la forma primaria de organizaciónmanifestación del lenguaje humano y unidad comunicativa de iteracción social". (p.17). Se concluye luego que las estrategias comunicativas para el desarrollo de la competencia comunicativa social de los alumnos debe girar, por eso, en torno del discurso. En la pág. 69 se concluye que para el desarrollo de la comunicación no es procedente el análisis morfológico o sintáctico de palabras $\mathbf{u}$ oraciones descon-

$\rightarrow$ textuadas y a veces artificiales, característico del enfoque gramaticalista, sino un análisis de discursos, de intercambios comunicativos reales, que relacionen con los actos de habla significados por medio de esas unidades. Respecto del papel de la enseñanza (cultivo) de la lengua materna en la escuela primaria y secundaria, conceptúan: "Se trata evidentemente de desarrollar esas competencias: pragmática, lingüisticas y comunicativa que ya los niños han adquirido. Pero desafortunadamente la escuela básica va en contravía. En lugar de elaborar acciones, interacciones y actividades comunicativas; en lugar de construir talleres, prácticas participativas para que los niños solucionen, o intenten solucionar, diferentes problemas del contexto escolar; en vez de elaborar procesos comunicativos que lleven a la escuela, a la comunidad; en lugar de todas estas actividades formativas y comuni- cativas, la enseñanza de la lengua materna, en la educación básica, se dedica a la transmisión de principios gramaticales abstractos que en la mayoría de las veces los mismo maestros no entienden". (p. 130)
Esta contrastación permanente pone en guardia saludablemente al lector; adolece eso sí de indiscriminación.

Por supuesto hay que decir que para otros menesteres los estudios formales son imprescindibles (desarrollo de las habilidades de pensar, por ejemplo), que en otros niveles educativos o para el logro de otros objetivos educacionales son básicos, e incluso que el tratamiento comunicativo generalmente supone el conocimiento gramatical.

Las tres orientaciones gramaticalistas postuladas por los autores corresponden en general a las de las programaciones curriculares del Ministerio de Educación que se han sucedido. $Y$ en verdad han encauzado en alguna medida la formación de los educadores, la produción de manuales y la enseñanza misma. Pero una evaluación menos somera e indiscriminada de las prácticas de enseñanza en distintas regiones, niveles educativos y modalidades de educación, dejan ver experiencias relacionadas de una $u$ otra manera con el enfoque propuesto por los autores, que se han ido abriendo camino, aún cuando no con el impacto deseble. Al respecto vale la pena revisar la monografía de grado Innovaciones en la enseñanza del español y la literatura en Colombia (1983 - 1993) de Doris Pérez y Marta Inés Romero (Universidad Nacional, 1997) también, mi escrito : La enseñanza del lenguaje : estudio bibliográfico selectivo. ${ }^{42}$

Podría realizarse un análisis detenido para precisar si entre las orientaciones pedagógicas y entre los estudios lingüisticos en mención tiene lugar una

42 Arboleda, Ruben, 1989, El conocimiento sobre la enseñanza del lenguaje: estudio bibliográfico selectivo, Bogotá, Centro de Investigaciones de la Universidad Pedagógica 
ruptura o más bien un ascenso; podría pensarse por ejemplo en que la insistencia permanente del enfoque semántico comunicativo en el desarrollo de las habilidades de comunicación sea un antecedente de la propuesta de los autores, que ésta trasciende gracias a los avances que entre una y otra han presentado los estudios comunicativos del lenguaje y al mayor dominio de estos estudios por parte de quienes elaboran. Podría pensarse en la clásica tipología de la oración según la intención del hablante: asertiva, imperativa, interrogativa, desiderativa, como un antecedente del estudio de los actos de habla. Pero en fin ese análisis desviaría ahora del propósito de la obra que es centrar la atención en la perspectiva comunicativa y en los desarrollos de la pragmática lingüistica y la teoría del discurso, no tenidos en cuenta antes con igual aplicación.

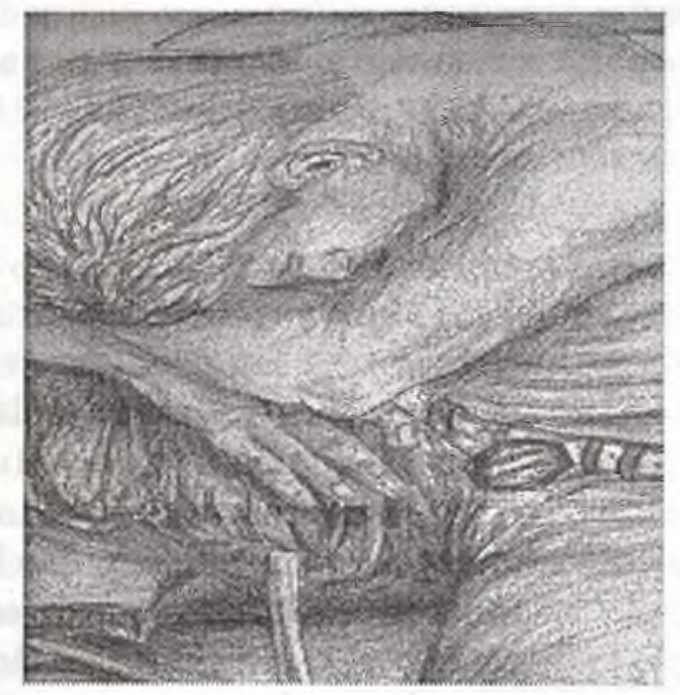

Los autores son optimistas respecto del logro de su objetivo. Caracterizan así un deber ser de la comunicación humana: "Dos personas se comunican, cuando en el proceso de la comunicación verbal. interactúan recíprocamer: : con el propósito de comprender, intercambiar y compartir: ideas, pensamientos, conocimientos, acciones, sucesos, emociones. deseos, vivencias, actitudes, creencias, ideologías e información sobre alguna realidad de interés social, con intencionalidad comunicativa de llegar a algún acuerdo, entendimiento, comprensión. propuesta de alternativas diferentes, ejecución de nuevos planes de acción, etc.; o simplemente, dar información significativa de algo a alguien". (p.38) Debe ser, por cuanto en el ser, las personas también nos comunicamos para engañar, confundir. desvirtuar, imponer un punto de vista, defender un interés individual, aprovecharse del interlocutor, etc.

Consideran que ese ideal "debería ser objetivado por todo tipo de comunicación masiva, audiovisual, cibernética y tecnologíaca". (p. 38). Y por supuesto. consideran que debería ser objetivado en la escuela, de tal manera que el discurso escolar se oriente "al conocimiento del mundo donde viven los niños, la comprensión de las interacciones sociales, la formación de la conciencia crítica y la participación en la construcción de una sociedad civil que les permita vivir de una manera más digna y más humana que la actual". (p.19) Sistematizan por eso unas perspectivas teóricas cuya asimilación rigurosa debe favorecer una acción coherente con ese ideal.

Y son optimistas respecto del papel de la educación, fundados en que si bien en los procesos de socialización tiene lugar una internalización del mundo social como una realidad congnitivo-significativa que determina las estructura de la conciencia del individuo, ahí no termina todo; por el contrario, la internallización subjetiva lo objetivo, provocando así nuevos procesos de externalización que potencian "la objetivación de nuevos roles más fuer:es y complejos para la creación, recreación. construcción y reconstrucción de un nuevo mundo social" (p. 49). 
Refuerzan la aserción con esta cita de Mead, quien por su parte refura a Dewey: "el individuo no es un esclavo de la sociedad. Constituye la sociedad tan ligítimamente como la sociedad constituye al individuo". Implícitamente se destaca en todo caso el arraigo y la fuerza de esas estructuras, de la conciencia y la no conciencia también, forjadas durante los primeros años de internalización del mundo social.

Pero el optimismo no les oculta las limitaciones para el logro del propósito derivadas de la acción educativa misma, cuando no sólo uno de los contextos inmediatos, la escuela, le es favorable, sino sobre todo el contexto sociocultural en el cual se inscribe, caracterizando por una honda crisis sociopolítica en todos los órdenes. Con razón afirman en la p. 229 : "si el contexto escolar se caracteriza por una comunicación vertical, autoritaria y un proceso de aprendizaje memorístico y dogmático, con toda seguridad los niños no se formarán una conciencia crítica productiva".

L Justamente por eso en un texto de 1989 capaz de controlar la museización (no hay por que colmillar esta creación verbal) de las experiencias, que desafortunadamente ya se ha registrado. Las experiencias de vanguardia tienen que expandirse progresivamente, afecta al grueso de educadores, tomar asiento en los centros de formación de docentes para que cada vez resistan mejor los embates

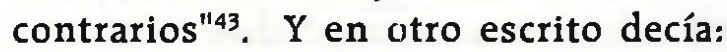
"Como nos podemos dar cuenta, el diseño de políticas educativas a una superación del fracaso escolar (repitencia, deserción) impone un acercamiento integral; cuestión sumamente compleja, debido a su dependencia de nuestra realidad socioeconómica. No quiere esto decir que planteemos la inutilidad de nuestros esfuerzos frente al mejoramiento de la pedagogía de la lengua (...) ni la inutilidad de las políticas del sistema educativo frente al fracaso escolar, sino la importancia de la captación de sus alcances reales y su vinculación a proyectos de superación integral a proyectos que conmueven la estructura de la escuela y la estructura social".44

Como se ha visto, la obra de Justo y María Teresa aporta elementos teóricos para fundamentar el ejercicio de una educación acorde con los desarrollos en las ciencias del lenguaje. Comparto y enfatizo con los autores la inconveniencia de una actitud muy extendida: considerar que la investigación teórica es ajena al educador. que lo pertinente para él es la aplicación. La sujerencia didáctica "lo práctico" (según se olle decir), como si el dominio de una teoría no resultara práctico, en el sentido de orientador, para la acción educativa. Es

43 Id.,p.41

44 Arboleda, Rubén, 1991, "Lengua escrita y rendimiento escolar", en la revista Forma y Función, Bogotá, Universidad Nacional, Departametno de Lingüística, No.5, 1991, p.98 
de esperar un acercamiento riguroso de los educadores a la obra, para evitar distorsiones como la que se ha registrado frente al llamado enfoque semántico-comunicativo: por falta de profundización, de capacitación del magisterio, ha sido frecuente el reenvasamiento improcedente del análisis semántico propuesto por las categorías morfosintácticas más conocidas. La confusión no se hizo esperar ${ }^{45}$. De otro lado, talvez no sobre advertir que, como se insinúa en el segundo capítulo, de ninguna manera se pretende que los conocimientos compendiados en el libro sean presentados como objeto de estudio en la educación básica primaria: lo que se busca es que dicho conocimiento inspire en los educadores las estrategias con las cuales se van a cultivar la competencia comunicativa social de los niños o, visto el asunto desde otro ángulo, sólidamente asimilado oriente la búsqueda de tales estrategias. El peligro advertido es real en los espacios educativos donde se trabaja más en la transmisión de contenidos que en el cultivo de la competencia comunicativa. Si así sucediera, el cambio se reducirá al de los contenidos reproducidos. Lo anterior no pretende significar que en otros niveles de la educación básica este conocimiento no pueda ser objeto de estudio en sí mismo.

A lo largo del libro se insiste en una orientación el discurso escolar hacia una interacción social comunicativa de calidad, hacia el conocimiento del mundo, la formación de la conciencia crítica, la participación en la construcción de una nueva sociedad civil, entre otros aspectos; complementariamente, y recurriendo a una idea nuclear e integradora de la ética del colega Leonel Arias, podría hablarse de una educación para el desarrollo humano. En este marco, los procesos educativos proyectados contemplarán sin duda el cultivo de la individualidad de los educandos, de sus percepciones particulares, de sus ensoñaciones, en fin, de la poeticidad, que es inherente a los miembros de la especie humana y se refleja en universos posibles como los de la muestra siguiente. Estos se desencadenaron o se habían desencadenado en opitas con quienes en un curso breve nos dedicamos a pensar la escritura autosignificativa en contraste con la transcriptivg; sin pretensiones artísticas. sólo de autorreconocimiento de una facultad nuestra.

\section{1}

En la noche oscura caminando insegura por caminos de herradura, reemplazar los míos por ojos de luciérnaga encendidos.

\section{Nelly Becerra}

\section{2}

Siento miedo a mi vejez. siento miedo a la oscuridad. siento miedo a las serpientes víboras que atan e inyectan veneno $y$ uno puede morir.

Siento miedo a la peste de la humanidad porque contagia, siento miedo, mucho miedo.

45 Ver: Arboleda, Rubén, 1987, "La Reforma Curricular: el caso de Español y Literatura", en Revista Colombiana de Educación, Bogotá, Centro de Investigaciones de la Universidad Pedagógica, No, 18. 
3

Mañana quisiera volar.

Volar por entre las nubes sin saber a donde parar, sin que existieran días, ni noches, ni horas, ni segundos, sólo volar y volar.

\section{Beatriz Cortés}

\section{4}

En una fiesta de pueblo se hacía la procesión de la virgen. Muchisima gente caminaba detrás de la imagen rezando y cantando, cuando de pronto veo a mi mamá vestida con un atuendo largo, color claro, que venía hacia mí. Caminé atónitamente a encontrarla, hubo un momento en que la tuve muy cerca y cuando pensé hablarle desapareció. Confundida la busqué entre la multitud. ¡No la encontré! Miré hacia atrás y la ví de lejos; seguía caminando. De pronto levantó su mano llamándome; sin vacilar me dirigi hacia ella por un

camino solitario y de poca vegetación, pero no permitió que me acercara, y de nuevo desapareció sin decir nada, dejándome con una inmensa nostalgia.

\section{María Esther Aguja}

5

Hay días en que me parezco a todo el mundo.

Al diablo por su limitación.

Al niño que inocente peca.

Al rico que nunca peca aunque lo haga. Al sol que abrasa la pasión de tu cuerpo. Al papa que quiere perdonar al enemigo.

Al guerrillero que mata la ilusión.

A la flor, a los rios, a los montes.

Al carbón, al diamante, al rubí.

Hoy me parezco a tí.

Aquileo Perdomo

6

Iba por un camino muy largo. De pronto me encontré con un hermoso árbol, en el cual había un nido con una paloma que dormía. Al sentir mis pasos salió volando

hacia lo alto. De repente se abrió su cuerpo y de alli salieron muchas palomitas

brillantes que también volaban por el cielo.

\section{Nohemi Parra}

En alguna medida estos universos posibles encarnan estructuras ancestrales de pensamiento que de una $\mathbf{u}$ otra forma orientan nuestro caminar por el mundo. 